\title{
The semantic side of decision making
}

\author{
DOUGLAS L. MEDIN, HILLARIE C. SCHWARTZ, \\ SERGEY V. BLOK, and LAWRENCE A. BIRNBAUM \\ Northwestern University, Evanston, Illinois
}

\begin{abstract}
The research reported in this paper follows the perspective that decision making is a meaningful act that conveys information. Furthermore, the potential meanings associated with decision options may affect the decisions themselves. This idea is examined in the contexts of compensation, donation, and exchange. In general, judgments were relation dependent and meaning dependent. Furthermore, the results show nonmonotonicities and limited substitutability in a pattern that challenges straightforward ways of mapping decisions onto a common currency of utility.
\end{abstract}

Human decision making is imbued with meaning. The decisions taken are semantically parsable to the person making them and can be sorted into distinct "kinds" or domains. They often convey information to others who are affected by or observe them, and they are used to draw attributions. Also, decision-making strategies are themselves potentially objects of meaningful discourse or reflection.

The focus of the present paper is on the influence of meaning on judgments concerning substitutability or exchangeability, as well as on attributions associated with decisions. We will show (and, as we shall see, other literature shows) that the meaning component of decisions gives rise to complexities that undermine attempts to use monetary value or straightforward notions of a "homogeneous currency" (e.g., subjective utility) as a close proxy or descriptive framework for judgments. We do not claim that utility theory is incapable of accounting for our results. One may always be able to assign a utility to a configuration of components associated with some context in a manner that conforms to judgments. Instead, our focus is on the incompatibility of meaning with desirable properties of a common scale of value or utility. That is, the form of utility theory that survives may be so weakened and distanced from empirical variables as to lose much of its explanatory and even descriptive value.

\section{Attributional Aspects of Decision Making \\ Decisions are often seen as conveying important attri- butional information about the values or even character of the person making them, and this is often taken into ac- count by the person making the decision. There is an ob- vious and perhaps trivial sense in which this must be true, of course. If a person chooses $x$ over $y$, then it seems true by definition that they prefer $x$ to $y$. But suppose that $x$ and}

\footnotetext{
This work was supported by NSF Grant BNS 95-11757 and NIH Grant MH55079. The authors thank David Messick for advice and encouragement in all phases of this research and Rob Goldstone for perceptive comments. Correspondence should be addressed to D. L. Medin, Northwestern University, 2029 Sheridan Rd., Evanston, IL 60208 (e-mail: medin@nwu.edu).
}

$y$ are seats at a restaurant table, and seat $y$ clearly has a better view and more leg room. Then, when a person who is on a date chooses $x$ over $y$, an alternative to the idea that they actually prefer $x$ is that they are being thoughtful or courteous. Or, if the person is in the company of an elderly person and chooses $y$ over $x$, one might think they are rude or disrespectful. The point is that people make attributions about the behaviors of others and that decisions may constitute particularly meaningful behaviors.

Indeed, decisions are often made with the explicit goal in mind of conveying information. For example, a person completely unhappy with his or her dining experience in a restaurant may choose to leave a 1-cent tip rather than no tip at all. The goal of leaving a 1-cent tip is to make sure that the message of dissatisfaction is conveyed, since leaving no tip could possibly be attributed by the waiter as forgetfulness or stinginess on the part of the customer. To verify this intuition, we asked 33 Northwestern University undergraduates to imagine they had a job waiting on tables in a restaurant; we then queried them as to whether they would rather receive no tip or a 1-cent tip. The overwhelming majority ( 29 out of 33 , highly significant by a binomial test) indicated that they would prefer no tip. Their comments indicated that a 1-cent tip would be perceived as an insult and a mean act. Note that these judgments imply a nonmonotone relation between monetary value and utility, where no tip and a large tip are preferred to a very small tip (we considered the assumption that a large tip would be preferred to no tip too obvious to test).

\section{Kinds of Decisions}

We also believe decisions are divisible into kinds based on the meaning they convey. Furthermore, there are both across- and within-kind obstacles to notions of common currency or simple exchangeability. For example, attempts to determine how much value people place on environmental goods (e.g., saving a lake from pollution) have been frustrated by the fact that respondents may treat the good as something that cannot be traded off for money (Kahneman \& Knetsch, 1992). In short, peo- 
ple may treat certain goods as linked to a moral, rather than an economic, decision. Baron and Spranca (1997) refer to such contexts as involving protected values.

Consider also the recent study of Tenbrunsel and Messick (in press), who presented research participants with a resource dilemma in which individual self-interest is in conflict with a cooperative solution that preserves the resource. In this situation, the decision context is ambiguous and subject to different construals. They found that mild sanctions for noncooperation actually increased noncooperation compared with no sanctions. Other measures suggested that, without sanctions, participants viewed the dilemma as involving a personal or ethical decision; with mild sanctions, participants tended to see the dilemma as a business decision (see also Bazerman, Tenbrunsel, \& Wade-Benzoni, 1998, and Larrick \& Blount, 1997, for related evidence).

To examine a protected value in the social domain, we recently asked 18 Northwestern University undergraduates to imagine that they were married and to make judgments about their willingness to trade their wedding ring for an identical ring plus various amounts of monetary compensation (up to $\$ 1$ million). Of the 18 participants, 16 indicated that no amount would suffice; the other 2 suggested they would trade for $\$ 100,000$ and $\$ 1$ million. This pattern of refusing to trade was reliably higher for a wedding ring than for a parallel scenario presented to 18 other undergraduates involving what was described only as a gold ring $\left[\chi^{2}(1)=9.8, p<.01\right]$.

Justifications for the wedding ring scenario reinforce the idea that symbolic value dwarfed monetary value. Some examples are the following: "It wouldn't matter if the ring my husband gave me was made of plastic"; "(To trade) would be a betrayal"; and "I think it is not a money issue but a meaning issue." When asked to imagine possible circumstances under which they would trade the wedding rings, participants often employed solutions (e.g., to enable the children to go to college; to pay for a needed operation) that integrated the symbolic value of the ring and commitment to the well-being of spouse and family. This example is far from counterintuitive, but it does serve to reinforce the idea that people resist assigning a monetary value to something that has symbolic value. Actually, this conclusion needs to be more nuanced. A lost, stolen, or accidentally damaged wedding ring would have very different implications than a ring exchanged for money. In other words, it is the meaning attached to a willingness to give up something of deep symbolic value that is critical.

Although the notion of kinds of decisions is fairly well established, it is difficult to provide a precise definition of kind. Meaning may depend on cultural conventions and inferences about goals even within a narrow domain. For example, we have found that, within the sphere of donations, attributions are not some straightforward function of monetary values. For example, in preliminary work, we asked Northwestern University undergraduates to rate their liking for a person who donated $\$ 500$ to Princeton
University. The person was described as being either a billionaire CEO or a shoe salesman. The shoe salesman received positive ratings $92 \%$ of the time compared with only $20 \%$ for the CEO (the CEO was also described by participants in very negative terms in an open-ended descriptive adjective task). Of course, these judgments could be revealing a general dislike for CEOs. However, when the $\mathrm{CEO}$ was described as donating a first edition of poems valued at $\$ 500$ to Princeton rather than cash, positive ratings for the CEO increased from $20 \%$ to $75 \%$. Our interpretation is that the cash donation was viewed relative to the CEO's wealth but that the book donation was seen as a different kind of act (see also Beattie \& Baron, 1995, Chapman, 1996, 1998, and Goldstein \& Weber, 1995, for further evidence on domains of decisions).

In a follow-up study, the multibillionaire scenario was described in two stories in which the final use of the donation was the same. Thirty-seven Northwestern University undergraduates rated the multibillionaire after reading one of two scenarios. In the first viguette, the donation is $\$ 500$ to the Princeton University Library, which is used to purchase books. In the second story, the multibillionaire finds the first edition of poems in an antique desk he has purchased at auction, is told that it is worth $\$ 500$, and then donates it to the Princeton University Library. The story continues that the library already has several first editions, and the head librarian sells the gift for $\$ 400$ and uses the proceeds to purchase books. Note that the latter scenario undermines the idea that the book had strong sentimental value for the multibillionaire or that the gift met a special need for the library. Note also that the final value for the library was less in the second vignette than in the first. Nonetheless, the multibillionaire was rated reliably more favorably after the second story than after the first $[t(35)=2.09, p<.05]$. Our interpretation is that the book donation suggests a different kind of intention than the money donation, and the latter evokes the multibillionaire's wealth as the context for evaluating the gift. In brief, it is the perceived intention that serves to determine attributions, not the value of the gift by itself.

\section{Semantics of Exchange}

By semantics of exchange, we mean that there are consensual rules or principles that determine what kinds of exchanges are appropriate and how they will be interpreted. Consider the behavior of two friends, A and B. Imagine that one day $\mathrm{B}$ gives $\mathrm{A}$ a small wooden elephant as a token of affection. That seems okay, but note that it would be odd for $B$ to give $A$ the cash equivalent of the cost of the elephant. Now imagine that a week later A gives B a mug or a small wooden owl. Either seems okay (to our intuitions), within the bounds of "like-kind reciprocity." But like kinds cannot be taken too literally. Suppose A gives $B$ a wooden elephant (different from the one that $B$ gave A) or two wooden elephants. Giving one elephant seems to imply something like "this sort of stuff is to your taste, not mine" (unless their friendship in- 
cluded a mutual interest in wooden elephants). Giving two wooden elephants seems to be a confrontational oneupmanship. There is also a temporal dimension to these sorts of exchanges. For most contexts, it would seem almost rude for A to reciprocate after only an hour; whereas after a month or so there may be nothing wrong with $A$ giving $\mathrm{B}$ a wooden elephant.

Our general approach has plenty of precedents, the closest of which is the Fiske and Tetlock (1997) social relation theory. They argue that there are four fundamental types of social relations and that different principles of exchange are appropriate to each of them. For example, most friendships are an example of what they call equality matching, where there is a loose reciprocity and money is an atypical medium of exchange. Very close relationships may constitute communal sharing, where even to monitor reciprocity would be inappropriate. In contrast, business exchanges embody market pricing, where exchanges are precisely monitored and money is the prototypical medium. The fourth type of relation, authority ranking, involves dominance as, for example, the relation between a queen and her subjects. In dominance relations, exchange may be asymmetrical (e.g., subordinates paying tribute). In short, according to Fiske and Tetlock, there is a semantics of exchange that varies as a function of type of relation. Although some forms of exchange may involve culture-specific conventions, the general rules of exchange for a given type of relation are held to be universal. Violations of such rules may lead to confusion and negative attributions.

To establish that these distinctions are shared, we constructed three scenarios in which one person helps another move from an old house to a new one. What varied across scenarios was the relationship between the mover and the person being helped. In the first scenario, a son is helping a father move; in the second, the helper is described as a close friend; and in the third, the helper is described as owning a moving business. The first should entail communal sharing, the second equality matching, and the third (though it is somewhat ambiguous) market pricing. Fifty-two Northwestern University undergraduates read one scenario (varied randomly across participants) and were asked to choose among monetary compensation, nonmonetary compensation (e.g., a gift, ordering pizza), or no compensation as appropriate in response to the help. The modal response was exactly as predicted by the Fiske and Tetlock framework: money for the person who owned a moving business, nonmonetary compensation for the close friend, and no compensation for the son helping his father [Pearson $\left.\chi^{2}(4)=30.5, p<.001\right]$. In short, the appropriate exchange for the moving scenario was relation specific.

\section{Relationship With Other Research}

Other work in decision making is also concordant with our overall framework. Research by Shafir and Tversky (1992) and Shafir, Simonson, and Tversky (1993) demon- strates that people often want to have reasons for their decisions, so much so that they will violate axioms of utility theory in their efforts to obtain information that will allow them to justify their choices. The work of Thaler and others (e.g., Thaler, 1985; Thaler \& Johnson, 1990) on mental accounting demonstrates that even money is not a homogeneous entity but is often broken down into budgets or mental accounts. These accounts resist exchange of money between them. Perhaps the best known example of mental accounting effects is Thaler's vignette of a person either losing a movie ticket that had cost $\$ 10$ versus losing $\$ 10$ and asking participants to indicate whether they would now pay $\$ 10$ to get into the theater. Although the situations are identical with respect to overall wealth, people are far more likely to say that they would buy a ticket after losing $\$ 10$ than after losing a ticket.

\section{The Present Research}

In the following sections, we describe further conditions in which participants made judgments about scenarios involving exchanges. The comparisons evaluated how the meaning associated with exchange affects both attributions about the decision maker and judgments of exchangeability itself. In these scenarios, "meaning" was varied on the basis of the history of the good and the use to which the good would be put (intended use). Participants were given a variety of scenarios that required them either to make attributions about the decision maker or to make decisions about their own willingness to trade a good (almost always for money). We expected that judgments would systematically deviate from the nominal market value of the good, with the direction of deviation depending on the particular meaning instantiated. To reiterate, none of our observations are impossible for utility theory to describe, but the assumptions needed for an adequate description may rob the theory of much of its explanatory power.

We used scenarios that focus on three factors that affect exchangeability. The first is sentimental value and the idea that people may be reluctant to convert sentimental value to monetary value. The second and related idea is that monetary value may work to undermine the meaning or attributions that might otherwise be associated with an exchange. The third factor is source-independent value. The desirability or exchangeability of some entity depends, more or less by definition, on its market value and not on how that value was achieved (its history). We shall show that, to the contrary, history may be quite important.

\section{METHOD}

\section{Participants}

Northwestern University undergraduates participated in this study for course credit. The scenarios described in this paper are part of a larger set of experiments. The number of participants completing the task varies by scenario. Sample size per version of each scenario was generally about 20 . Exact sample sizes are provided in the tables in the Results section. 


\section{Materials}

Three scenarios will be discussed, each of which consisted of two or more versions. Each participant read only one version of every scenario.

The first scenario ("Land") was presented as follows:

You live on a plot of land with a market value of $\$ 100,000$. The land has been in your family for $[2 / 100]$ years. The developer approaches you and tells you about a piece of land not too far off that is extremely similar to the one you currently live on. An independent appraiser tells you that the new piece of land is valued at $\$ 100,000$. The developer asks you to move to this new piece of land and agrees to cover all expenses associated with the move.

There were two variables tested in this scenario. One variable was history of the land (whether the land has been in "your family" for 2 years vs. 100 years). The second variable was intended use (whether the developer planned to use your land to build a shopping mall vs. to build a children's hospital).

One dependent variable was the amount of incentive our participants needed to part with their land. The following interval scale was utilized (the participants were asked to check only one box):

$\quad \$ 0$ (will trade for nothing)
$-\$ 10$
$-\$ 100$
$-\$ 1000$
$-\$ 10,000$
$\quad \$ 100,000$
$-\$ 1$ million
- No incentive is high enough to trade

The participants were also asked to rate how they felt about the developer and about moving. A rating scale ranging from 0 (extreme negative feelings) to 9 (highly positive feelings) was used to measure both feelings about the developer and one's own feelings about moving.

In the second scenario ("Reggie"), we asked the participants to consider the following:

James had owned his terrier, Reggie, for five years. Reggie was a puppy when James bought him for $\$ 150$. One day when James and Reggie were walking in the park. they met a man who liked Reggie so much that he offered James $\$ 1,000$ for Reggie.

There were four versions of this scenario. A $2 \times 2$ design was used, in which one variable was the reason given by the man for wanting to buy Reggie (to serve as a companion for sick children at a Hospice vs. no explanation given.) James's response was the second variable (give Reggie away without monetary compensation vs. agree to sell Reggie for $\$ 1000$ ). The participants were asked to assess the extent to which they liked James (on a 0 -9 likeability rating scale). The participants were also asked to explain their rating.

The third scenario ("Bookstore") involved the first edition of a book and had two versions that are of interest to the present paper. The description was as follows:

Suppose you have a first edition of a book published in 1930 that is in excellent shape. A guidebook tells you that first editions in such excellent shape are worth $\$ 300$. You know from a reliable source that the book was once owned by [Adolf Hitler/the local bookstore]. A book collector approaches you and offers to buy the book from you. In order to convince you he offers money over the market value of $\$ 300$ as an incentive. How much incentive do you need?

The participants were offered the eight options described above, ranging from $\$ 0$ to $\$ 1$ million dollars and including the possible suggestion that no incentive would be high enough.

\section{Procedure}

The participants were given a booklet consisting of these and other scenarios (there were generally eight scenarios per booklet). Most participants finished the task in less than $30 \mathrm{~min}$.

\section{RESULTS}

\section{Scenario 1: "Land"}

In this scenario, we examined the effect of decisions on attributions about the self. Recall that we asked our participants whether they would trade their land for a similar lot close by. Sentimental value was manipulated by varying the length of time for which the land had been in the family's possession ( 2 years vs. 100 years). Intended use of the land (children's hospital vs. shopping mall) was also varied. The main dependent variable was willingness to trade the land. For purposes of analysis, we treated exchange ratings as an 8-point scale $(0-7)$ on which refusal to trade was scored as a 7 . We also report refusal to trade as a categorical response. Note that it was made clear in the scenario that the incentive money was money over and above the actual costs of the move.

The results from the Land scenario are summarized in Table 1. Willingness to trade depended on both period of possession (history) and intended use (hospital vs. mall). Not surprisingly, there was a main effect of history $[F(1,96)=8.07, p<.01]$. In the 2 -year condition, the average incentive level needed to trade was $4.7(S D=2.0)$ or corresponding to about $\$ 100,000$, with $20 \%$ indicating that they would never trade the land. In the 100-year condition, the average incentive level was $5.8(S D=1.6)$, roughly corresponding to $\$ 1$ million. Forty-five percent of the students in the 100-year condition indicated that no amount of incentive would be high enough to trade. In short, the participants were reluctant to exchange something of sentimental value for money.

Also of interest is the fact that there was a main effect of the intended use of the land $[F(1,96)=17.09, p<$ $.001]$. In the hospital condition, the average incentive level was $5.2(S D=2.3$; or about $\$ 100,000)$. When a shopping mall was to be built on the land, the average incentive level was $5.9(S D=1.0$; or approximately $\$ 1$ million $)$. Also, the percentage of participants who indicated that they would never trade the land was greater in the mall condition than in the hospital condition $(38 \%$ and $27 \%$, respectively).

This effect of history or symbolic value is illustrated by the following comments from the participants: "The land doesn't have much sentimental value to me" (2-year condition); "The land has been in my family's possession for 100 years, there are a lot of memories there" (100year condition). The participants' justifications of their choices showed that intended use of their land also played a powerful role in tempering concerns over sentimentality or increasing its salience: "The children's hospital makes me more willing to move, although it's hard because the land has been in the family for so long" (hospital condition); "I'd feel like I had sold out to the bad guys," and "I don't want to see my memories turned into a shopping mall" (mall condition).

When asked how they would feel about moving from their land, the participants felt significantly more positive $[F(1,95)=4.75, p<.05]$ if the land was going to be used to build the hospital $(M=5.0, S D=2.8)$ rather than used 
Table 1

Results From the "Land" Scenario

\begin{tabular}{|c|c|c|c|c|c|c|}
\hline \multirow[b]{3}{*}{ Land to be used for... } & \multicolumn{6}{|c|}{ Land in the Family for... } \\
\hline & \multicolumn{3}{|c|}{2 years } & \multicolumn{3}{|c|}{100 years } \\
\hline & $M$ & $S D$ & $n$ & $M$ & $S D$ & $n$ \\
\hline & \multicolumn{6}{|c|}{ Incentive Level Required for Trade* } \\
\hline Shopping mall & 5.4 & 1.0 & 25 & 6.3 & 0.8 & 30 \\
\hline \multirow[t]{2}{*}{ Children's hospital } & 4.0 & 2.5 & 24 & 5.0 & 2.1 & 21 \\
\hline & \multicolumn{6}{|c|}{ Feelings About Trade $\dagger$} \\
\hline Shopping mall & 4.8 & 2.8 & 25 & 2.9 & 2.6 & 29 \\
\hline \multirow[t]{2}{*}{ Children's hospital } & 4.8 & 2.6 & 24 & 5.2 & 2.9 & 21 \\
\hline & \multicolumn{6}{|c|}{ Feelings About Developer $\dagger$} \\
\hline Shopping mall & 4.6 & 2.3 & 25 & 3.9 & 2.1 & 30 \\
\hline Children's hospital & 5.5 & 1.9 & 24 & 6.1 & 1.6 & 21 \\
\hline
\end{tabular}

*The higher the rating, the less willingness to trade (scale ranged from 0 to 7 ). The higher the rating, the more positive the feelings (scale ranged from 0 to 9 ).

to build the mall $(M=3.7, S D=2.8)$. Although there was no main effect of history on ratings of how the participants would feel about moving $\left[F(1,95)=1.96, M S_{\mathrm{e}}=7.42\right.$, $p>.05]$, there was an interaction of use of the land and history $[F(1,95)=4.19, p<.05]$. The participants in the mall/100-year condition rated their feelings about the move much more negatively than everyone else. In short, these judgments did not simply reflect the general desirability or undesirability of a mall versus a hospital but negative feelings specific to the sentimental value (100year condition).

Some of the concerns about trading the land for a mall when it was in the family for 100 years can be summarized by these comments: "I'd feel terrible-like I was trading my family for money"; "I would feel guilty. I would be uncomfortable every time I went by the mall." The participants in the hospital conditions, on the other hand, tended to balance the loss of sentimental value with doing a "greater good": "I would be sad to sell my family's land but ... I am being selfless and generous for the children of the hospital"; "I would feel as if I had contributed to the success of the hospital and had done a good deed for humanity."

The intended use of the land also had a significant impact on the participants' ratings of the developer $[F(1,96)=$ $14.04, p<.01]$. As might be expected from the findings above, the participants rated their liking of the developer as much lower if he had approached them about building a mall $(M=4.2, S D=2.2)$ than if he had approached them about building a hospital $(M=5.8, S D=1.8)$. The history of the land had no impact on ratings of the developer $(p>.05)$.

\section{Scenario 2: "Reggie"}

This scenario was also designed to examine the effect of decisions on attributions, but the dependent variable was attributions about someone else who is making a decision, not attributions about the self. Recall that James was approached by a stranger and asked to give up his dog Reggie. We manipulated James's action (sell vs. give away) and whether the stranger's goals were charitable (hospice) or unmentioned (see Table 2). Of particular interest were the effects of James's receiving money for his dog.

As Table 2 indicates, the ratings of the owner depended on both why the man wanted Reggie and whether James took money in exchange. James was rated more favorably when he decided to give his pet away to a hospice than when he gave up his dog without an overtly stated reason ( $M=5.0, S D=3.1$, and $M=3.5, S D=2.5$, respectively) $[F(1,64)=5.75, p<.05]$. In addition, the participants rated James more favorably when he chose to give the dog away $(M=6.3, S D=2.5)$ rather than sell Reggie $(M=2.4, S D=1.9)[F(1,64)=54.47, p<.01]$. There was a directional, but not significant, interaction between the action that James takes (sell vs. give away) and the purpose of giving the dog away (hospice, unspecified) $\left[F(1,64)=3.22, M S_{\mathrm{e}}=4.37, p=.08\right]$. For the sell condition, it did not seem to matter much to the participants whether James was selling the dog to a hospice or selling the dog to a man for an unexplained purpose. In either case, the participants gave James very low ratings (about 2 on a $0-9$ likeability scale). However, within the give condition, the participants were much more favorable to James when Reggie was given to a hospice than when Reggie was given away for an unexplained purpose. In summary, taking money for the dog appeared to have almost completely undermined any favorable impression associated with the man's dog going to the hospice.

Protocol analysis showed that, in the give/hospice condition, the reactions were characterized by general approval with a tinge of apprehension about Reggie's welfare: "It was very kind to give his dog away to a charity ... but he might not have thought about the dog's best interests." In the give/unexplained condition, justifications for likeability ratings revealed confusion, ambivalence, and a desire to know the intended use. A typical response was, "I don't dislike or admire James. He did refuse the money which was admirable, but he just gave the dog away after having owned him for five years."

In the sell/hospice and sell/unexplained conditions, the participants were alarmed by James's willingness to part with Reggie in exchange for monetary compensation. Even the charitable intended use of the hospice did not help attenuate these feelings of apprehension: "By placing a monetary value on a companion of five years, James shows himself to be less concerned with relation-

Table 2

Likeability Ratings (on a 0-9 Scale) of James in the "Reggie" Scenario

\begin{tabular}{|c|c|c|c|c|c|c|c|c|}
\hline \multirow[b]{3}{*}{ Recipient } & \multicolumn{8}{|c|}{ Action } \\
\hline & \multicolumn{4}{|c|}{ Give } & \multicolumn{4}{|c|}{ Sell } \\
\hline & Median & $M$ & $S D$ & $n$ & Median & $M$ & $S D$ & $n$ \\
\hline Hospice & 8 & 7.2 & 1.8 & 19 & 2 & 2.5 & 2.3 & 17 \\
\hline Control & 5 & 5.1 & 2.8 & 14 & 2 & 2.2 & 1.4 & 18 \\
\hline
\end{tabular}

Note-The higher the rating, the higher the perceived likeability. 
ships... than with financial gain"; "I'm not too crazy about James treating his dog like a piece of merchandise"; "A pet is like a ... best friend, you don't go around thinking you can sell your best friend and buy a new one."

\section{Scenario 3: "Bookstore"}

Recall that the third scenario involved the first edition of a book once owned either by a local bookstore or by Adolf Hitler. The fact that Hitler once owned the book should make it more valuable. Indeed, about one third of the participants who read the Hitler scenario placed a high exchange value on the book and justified their reserve price in terms of its historical interest. No participant suggested that a book previously owned by Hitler would be less valuable by virtue of Hitler's notoriety. Yet the median reserve price $(\$ 1000)$ did not vary across scenario type. Perhaps most telling were the relative numbers of participants whose reserve price was $\$ 0$ ( 5 out of 26 for the Hitler case vs. 1 out of 25 for the bookstore) or who refused to set a price on the grounds that they would not want to profit from this transaction ( 4 vs. 0 , respectively). Overall, the participants were more willing to profit from the first edition owned by the bookstore than from the first edition whose value had been enhanced by its association with Hitler ( $p=.023$, by Fisher's exact test, two-tailed). In short, perceived value was not the sole determinant of the participants' principles of exchange.

\section{DISCUSSION}

Our observations demonstrate that meaning influences both judgments and attributions. Although these observations do not violate utility theory, they undermine the most natural descriptive framework for mapping a common scale onto choices. For example, the preference for no tip over a l-cent tip violates monotonicity on a scale of monetary value. The scenario involving a book once owned by Hitler shows that sellers may care about the source of value (and may prefer not to profit by it) (see Loewenstein \& Issacharoff, 1994, for a related example of source dependence). For exchanges such as someone helping another person move, the appropriate form of compensation depends very much on who the parties are. Money is an appropriate exchange only for the right relationships and only for certain kinds of exchanges. Conversely, nonmonetary goods and services are appropriate in certain contexts in which only money will do.

We believe that these considerations carry over to transactions as prototypical as buying or selling a used car-that is, it probably matters whether the two parties are strangers, close friends, or part of the same nuclear family. For example, the "the more the better" principle would likely hold only for strangers (if then). The scenario involving the multibillionaire making a donation of money or a book demonstrates that the perceived significance of an act (and even its polarity) depends not simply on its monetary value but on what kind of act it is perceived to be. Finally, the example of Reggie the dog indicates that
(1) people believe that things of sentimental/emotional value should not be sold (or given away), (2) intended use has a strong effect on attributions (when the dog would help sick children at the hospice, people made very favorable attributions about the owner), and (3) receiving money can completely undermine effects of intended use on attributions. We suspect that the negative attributions about the dog owner who gave up his dog for the hospice but received money for it would reverse if the scenario were continued by the owner's donating the proceeds to an animal shelter. What is critical to the attributions by others (and perhaps to self-attributions) is neither the act nor the material components associated with it but rather the presumed reasons for it (see Ahn \& Bailenson, 1996, for an analysis of the role of reasons in causal attributions).

Although most of our examples used money, we believe that there are serious limitations with trying to map value onto any homogeneous entity. As we have noted, there are other examples in the literature that make closely related points. For example, Sen (1985) describes a hypothetical situation of a doctor facing two critically ill people but having only one unit of medicine. A full unit is needed for the medicine to have any chance of working. If the unit is given to Patient $A$, there is a $95 \%$ chance it will work, and, for Patient B, there is a $90 \%$ chance of a cure. Forced to choose, the doctor would justifiably give the medicine to Patient A. But Sen raises the question of whether the doctor would prefer the option of a 50-50 chance mechanism to determine who would get the medicine. It is very plausible that the doctor would do so, apparently violating axioms of rationality (if you prefer Outcome A to Outcome B, then you should prefer a $100 \%$ chance of Outcome $A$ over a $50 \%$ chance for $A$ and a $50 \%$ chance for $B$ ).

Of course, one could circumvent this apparent violation by assuming that there is some utility associated with the doctor not having to make a decision that, in effect, condemns a specific patient to death. Note that, in adding this assumption, one is robbing utility theories of much of their power. We believe that, in the face of these sorts of retreats, it will prove more efficacious for analyses of decision making to include theories that explicitly address the role of meaning in choice and exchange. Two approaches that begin to do so are Fiske and Tetlock's relational model and Thaler's notions of mental accountiny. We take them up in turn.

\section{Exchangeability and DM}

Our findings suggest that meaning matters in understanding people's notions of exchangeability. But, what factors may play a role in determining what is exchangeable? One useful framework to think about these issues is Fiske and Tetlock's relational model of social interaction. Fiske and Tetlock's main argument is that exchanges between entities in different domains are often painful, condemned as unethical, or just plain taboo. Parents selling their children into slavery is an extreme example of the forbidden mixture between community sharing and mar- 
ket pricing. Other cross-domain tradeoffs are less offensive and may only be perceived as tactless. For example, offering money to a neighbor who helped you with a spare tire is not immoral, but it may be seen as a display of bad taste. In general, even contemplating a cross-domain tradeoff jeopardizes the integrity of the types of relationships considered.

One might suggest that Fiske and Tetlock's domain of community sharing, a mode of exchange that involves unlimited sharing of resources, roughly corresponds to a kind of domain of moral goods. Since transactions within a domain are hypothesized by Fiske and Tetlock to be relatively easy, it is reasonable to hypothesize that a given moral good may be at least somewhat substitutable for another moral good. This may suggest that, in our "Land" scenario, the tradeoff of family land for a children's hospital will be judged as much more palatable than a tradeoff involving a shopping mall. We are suggesting that, although each of the scenarios set up a context for an exchange based on market pricing, additional factors inevitably come into play. Specifically, the entities involved are infused with additional meanings and tied to other kinds of valuation.

Although the Fiske and Tetlock framework is useful in thinking about exchanges, we believe that the particular meanings associated with decisions and exchanges are more precise mediators of action. Consider again Reggie the dog. Imagine that Reggie's owner met another dog owner and that the latter suggested trading dogs (with or without some monetary bonus thrown in). Suppose that, in addition, the dog owners were good friends. Our intuition is that people would nonetheless have negative attitudes about dog swapping because sentimental value, loyalty, and the like are specific to the particular individuals involved. In the same vein, it would be very strange indeed for two friends, each married, to exchange wedding rings with each other. One can, of course, imagine other cultures that would attach different meanings to such kinds of exchanges, but, at least in our culture, sentimental values are not interchangeable.

Note that, in the above examples, sentimental value involves not just the owner but other sentient entities (ancestors and relatives, one's spouse, one's dog). Part of the meaning of objects of sentimental value may be in the form of obligations to others. Exchange principles may be different for entities for which the only meaning factor is the relation of the person to the entity (e.g., a scarf that one has knitted). We believe that, in cases such as this, the exchange principles may be different because meanings may be different, not because meaning is not involved. These sorts of possibilities remain to be explored empirically.

\section{Meaning and Mental Accounting}

We were particularly struck by our participants' appeal to "solutions" involving the specific, meaning-bearing categories containing the objects of their decisions when asked to justify those decisions or provide a context in which a particular decision would seem acceptable to them. Thus, when asked to consider the grounds for selling a wedding ring, many participants offered family needs of various kinds (e.g:, medical) as a possible justification. The notion of naming a wing of the hospital after the family if family land had to be sold to build it had a similar flavor.

One way to approach these issues is to consider the inherent functions of the particular categories or domains of objects and decisions in question, as well as of forming and using such categories in general. In the case of the land, for example, the "family homestead" serves a number of purposes: It is a memorial to the past, it serves as a place to make new family memories, it ties the generations together, and so on. If we consider now the notion of a hospital wing named after the family, it serves one of these purposes, memorial (in fact, in a more public way than the land itself), but not the others.

Although such explanations are not too difficult to generate (indeed, we expect that they can be found for many, if not all, instances of this sort) and are quite plausible, we believe they do reveal something genuine about the criteria people are applying in making these decisions; what they do not explain is why it matters, its functionality. In other words, why do the category boundaries seem so rigid, and why should tradeoffs between categories be so much harder than exchanges within them?

In this context, the notion of "mental accounting" developed by Thaler and others seems quite relevant. The question is, what is the function of mental "accounts," and why do they have the properties that they do? We think a good way to approach this problem is to simply cross out the word mental, and ask, what is the function of accounting, and why do accounts have the properties that they do?

We speculate that accounting exists for a number of reasons. First, it is a way of keeping track of expenditures and returns over time. Companies and individuals need to know where value is coming from and where it is going. In the absence of this information, it would be difficult to make rational decisions about resource allocations.

Second, accounting (more exactly, budgeting) enables hierarchical decision making. Having decided the relative benefits of home ownership, recreation, education, and so forth, people can allocate resources to these categories in the abstract, without needing to consider the specific choices involved in each category. This serves two purposes. One is that it enables "strategic" decision making to take place without the distraction of the myriad of details involved in actually carrying out the strategies. The other is that it facilitates "tactical" operational decision making by rendering the comparison set of options manageable, and indeed commensurable, because they fall into a given strategic category. Thus, confronted with a recreational choice, we are not required to think of all the other things we might do with the time and money they entail, only about other recreational options. In short, accounting makes computational sense.

Finally, accounting exists to enable learning. When something negative happens, it is important to trace the cause back to the appropriate factors and decisions, so 
that they can be considered and made differently in the future. To take a variation of Thaler's example: If you go to a show, and you do not like it, then the failure probably lies in your tactical decision making about how to spend your leisure time and money, not in your overall strategy of allocating a certain amount of these resources to leisure activities. We are suggesting that the "pain" (or cost) of a failure must accrue to the category in order to learn decision rules or expected utilities within that category. Assigning the cost to a higher category, in contrast, will trigger learning about how to allocate resources more strategically. Again, these ideas remain to be explored.

\section{Conclusions}

We have argued that human decision making is infused with meaning. Decisions are meaningful to the person making them, and they often convey meaning to others who are affected by or observe them, sometimes intentionally so. This suggests that taking a semantic approach to human decision making can yield some insights into decision-making operation that might not lie within the range of other approaches. We end with a quotation that nicely summarizes our thesis:

The meanings elaborated in decision making have importance beyond the mundane realities of rendering decisions. Decision making and the activities surrounding it have considerable symbolic importance. In the course of making decisions, decision makers develop and communicate meaning not only about decisions but also more generally about truth, about what is happening in the world and why it is happening. They define what is morally important and what is proper behavior. They elaborate a language of understanding and describe how actions are properly explained and justified. (March, 1994, p. 212)

\section{REFERENCES}

AHN, W. K. \& BAILENSON, J. (1996). Causal attribution as a search for underlying mechanisms: An explanation of the conjunction fallacy and the discounting principle. Cognitive Psychology, 31, 82-123.

Baron, J., \& Spranca, M. (1997). Protected values. Organizational Behavior \& Human Decision Processes, 70, 1-16.
Bazerman, M. H., Tenbrunsel, A. E., \& Wade-Benzoni, K. A. (1998). Negotiating with yourself and losing: Understanding and managing conflicting internal preferences. Academy of Management Review, 23, 225-241.

BEATTIE, J., \& BARON, J. (1995). In-kind and out-of-kind penalties: Preferences and valuation. Journal of Experimental Psychology: Applied, 1, 136-151.

Chapman, G. B. (1996). Temporal discounting and utility for health and money. Journal of Experimental Psychology: Learning, Memory, \& Cognition, 21, 771-779.

Chapman, G. B. (1998), Sooner or later: The psychology of intertemporal choice. In D. L. Medin (Ed.), The psychology of learning and motivation (Vol. 38, pp. 83-113). San Diego: Academic Press.

Fiske, A. P., \& TeTlock, P. E. (1997). Taboo tradeoffs: Reactions to transactions that transgress the spheres of justice. Political Psychology, 18, 255-297.

Goldstern, W. M. \& Weber, E. U. (1995). Content and discontent: Indications and implications of domain specificity in preferential decision making. In J. R. Busemeyer, R. Hastie, \& D. L. Medin (Eds.), The psychology of learning and motivation (Vol. 32, pp. 83-136). San Diego: Academic Press

Kahneman, D., \& KNETSCh, J. L. (1992). Valuing public goods: The purchase of moral satisfaction. Journal of Environmental Economics \& Management, 22, 57-70.

LARrick, R. P., \& Blount, S. (1997). The claiming effect: Why players are more generous in social dilemmas than in ultimatum games. Journal of Personality \& Social Psychology, 72, 810-825.

LoewEnstein, G., \& IssacharofF, S. (1994). Source dependence in the valuation of objects. Journal of Behavioral Decision Making, 7, 157 168.

MARCH, J. G. (1994). A primer on decision making. New York: Free Press.

SEN, A. (1985). Rationality and uncertainty. Theory \& Decision, 18, 109-127.

Shafir, E., Simonson, I., \& TVersky, A. (1993). Reason-based choice. Cognition, 49, 11-36.

Shafir, E., \& TVERSKY, A. (1992). Thinking through uncertainty: Nonconsequentialist reasoning and choice. Cognitive Psychology, 24. $449-474$

Tenbrunsel, A., \& Messick, D. E. (in press). Sanctioning systems, decision frames, and cooperation. Administrative Science Quarterly.

THALER, R. H. (1985). Mental accounting and consumer choice. Marketing Science, 4, 199-214.

ThALER, R. H., \& JOHNSON, E. J. (1990). Mental accounting and consumer choice. Management Science, 36, 643-660.

(Manuscript received July 16, 1998; revision accepted for publication March $11,1999$. 